\title{
Deep Breathing Alters Visual Motion Perception
}

\author{
Ahmad Yousef ${ }^{1}$ \\ ${ }^{1}$ School of Computational Science and Engineering, McMaster University, Hamilton, Ontario, Canada \\ *Correspondence: mohamas2@mcmaster.ca
}

\begin{abstract}
This article is to provide evidence that deep breathing had great influence on the perception of stimuli that trigger illusory motion perception. We had used two different stimuli; the first one can be considered as bistable rivalrous stimulus because it can trigger illusory motion reversals during its motion. The second stimulus is stationary, namely rotating snakes illusion, it is also bistable rivalrous stimulus because it has two states, stationary versus illusory motion. We had noticed that deep inhalation slows down the speed of the first stimulus and eliminates the illusory motion perception of the second stimulus. This might be because the amount of the hemoglobin red blood cells, possibly including the rich oxygenated ones, might be forcibly reduced in the brain during the intended inhalation, in turn, different parts in the brain, including hMT+ region, might be partially deactivated, see reference 1 and 2. Significant reduction against stimulus' contrast is known to slow down the perceived speed, it also diminishes the activities of the retinal peripheries and their corresponding neurological connections that collectively build up the peripheral brain; we therefore suspect the peripheral $\mathrm{hMT}+$ region to be inactivated by the deep inhalation. Strong exhalation, however, triggers illusory motion reversal for the first stimulus, and promotes illusory motion perception for the second stimulus; behavior that can be explained by the increased amount of the hemoglobin red blood cells that may activate different necessary regions in the peripheral brain.

Astonishingly, we found that deep inhalation and exhalation sufficiently can control the aforementioned bistable visual perception.

Introduction

Respiratory sinus arrhythmia is an accommodation process which occurs during breathing cycles; namely the heart rate accelerates for the inspiration and decreases for expiration, see references 3 , and 4 . Naturally, with the assistance of the heart, the lungs pull the deoxygenated hemoglobin red blood cells for purification purposes, namely, switch the deoxygenated hemoglobin red blood cells into oxygenated hemoglobin red blood cells. Deep
\end{abstract}

inhalation, which requires human attention, however, seems to indistinguishably suck both of deoxygenated and oxygenated hemoglobin red blood cells from every organ in the human body including but not limited to the brain; possibly leading to temporal significant reduction of the oxygenated hemoglobin in the brain,

and thus, the visual perception is altered. In the presence of dilated pupils, preliminary studies showed us that deep breathing can control the rivalry of stimulus 1, a stimulus that triggers rivalrous perceptions between actual direction of movement and illusory motion reversals; in another word, deep inhalation significantly slows down the speed of motion, while deep exhalation not only accelerates the perceptual speed but it triggers illusory motion reversal, see experiment 1 at reference 5. We had previously showed that retinal peripheries can sufficiently generate illusory motion reversals, see reference 6 . More importantly, reduction against the stimulus' contrast suppresses the illusory motion reversals, see reference 7. Important to emphasize, the aforementioned observations demonstrate that breathing can sufficiently govern the aforementioned perceptual rivalry of motion directionalities. The primarily investigations had also concluded that at the end of deep inhalation; the illusory motion of a rotating snakes' stimulus can be entirely suppressed, however, at the end of a deep exhalation, the illusory motion can be high promoted, see reference 5 , and 8 . Collectively, the aforementioned facts might grant evidence that the retinal peripheries and their neurological connections that build up the peripheral brain are relatively inactive through inhalation, and relatively active through exhalation!

Materials \& Methods

Twelve human subjects with dilated pupils were recruited to participate in this study. Two experiments have to be tested; each for them has two sections, reference section, and breathing section, see reference 5. In experiment 1, the stimulus subtends 12.9 DVA length and 1.37 DVA width and consisted of 9 cycles, and runs at constant speed of 12.9 DVA/sec. Each 
human subject has to experience the experiment for three times for data finetuning. In the beginning of experiment 1 , any human subject who cannot see illusory motion reversals must be excluded; however, since we had chosen all of the subjects with dilated pupils; all of them were able to see the illusory motion reversal from the first time. In the reference section of experiment 1 ,

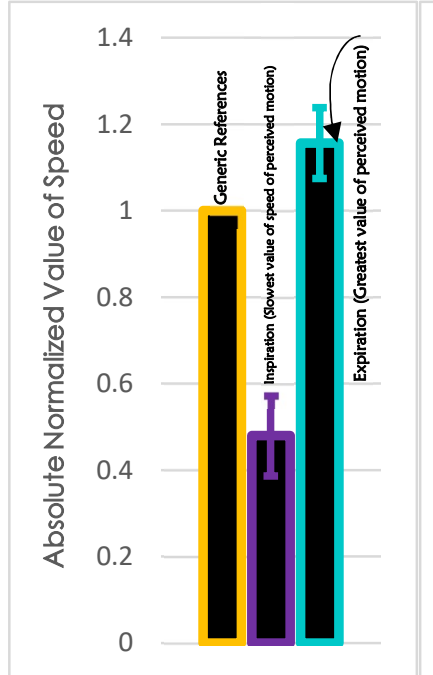

Collected data for experiment 1 while they are centrally focus their attention on signal cyclic element that builds the entire stimulus, human subjects are requested to generally estimate the ratio between the speeds of the real forward motion and the illusory backward motion; these estimations will be used for quantifying the influence of breathing on the visual motion perception. In the breathing section of experiment 1 , the human subjects have to follow certain protocol, namely, take a deep inhalation, execute a profound exhalation, take an extremely very short break, and then repeat the cycle until the end of the trial. Subjects are not instructed to shorten or elongate their breathing cycles, we kept them to choose their own comfortable timing; however, we were strict about 'deep breathing'. Each human subject is requested to report the influence of inhalation or exhalation on the aforementioned stimulus and to report the slowest speed for inhalation, and the greatest speed for exhalation compared with the generic references. Astoundingly, all of the human subjects had noticed the stimulus to move much slower in the correct direction during deep inhalation, however, it moves a little bit faster and in the illusory direction during profound exhalation. In experiment 2 , the same protocol of the breathing cycles had been used, however, we had used a fixed frame of reference, see reference 5 . Unlike experiment 1 , we had asked the human subjects to report the strength of the illusory motion at the end of inhalation and the end of exhalation processes. Astonishingly, all of the human subjects had not seen any illusory motion at the end of each inhalation, however, some of them had seen significant illusory motion even beyond the reference level at the end of exhalation. Based on the

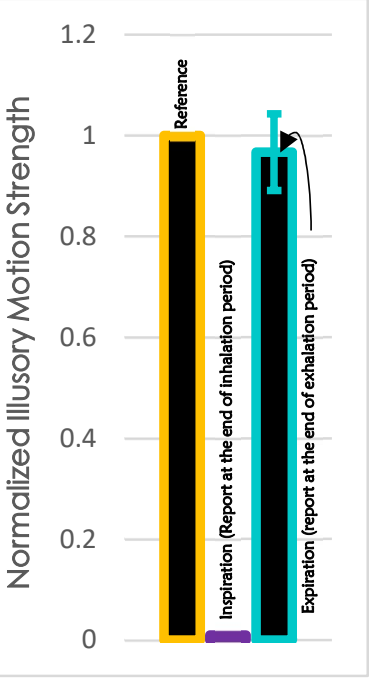

Collected data for experiment 2 previously mentioned frame of references, the behavioral data are collected; and for each human subject, the data were simply averaged to just render one mean value, these mean values are then used to estimate the simplest statistical values among all of the subjects, namely, the average and the standard error of the mean. In a previous study, we had successfully deactivated the peripheral fusiform face area, one of the two distinct fusiform face areas see reference 1. We were able to achieve outstanding inactivation to the aforementioned area through manipulations to the visual stimulus, such that V4 and hMT+ will request for greater amount of oxygenated hemoglobin, in turn, the peripheral FFA will not be receiving enough oxygenated hemoglobin, and therefore, the rabbit face will be vanished from the visual awareness. Closer technique had been used in this study, however; the temporal reduction of oxygenated hemoglobin had been achieved by deep inhalation. As shown in the upcentral infographic, there is significant correlation between deep inhalation and declaration of the perceived speed, for both moving or static stimuli, as if deep inhalation reduces the amount of the rich-oxygen blood in the brain though possibly disallowing important peripheral parts of $\mathrm{hMT}+$ region form working properly; this might be because the lungs had sucked larger amount of the red blood cells from the brain. In the exhalation process, however, the heart pushes huge amount of oxygenated red blood cells to the brain; allowing the peripheral areas in the hMT+ region to work beyond the limits, and therefore perception of illusory motion. Lastly and most importantly, deep inhalation does not only slow the perception of motion, but it might intensify the sympathetic nervous system and temporally increase the heart rate; similar to what might be happening in frightening events. The aforementioned hypothesis might possibly object what was offered in reference 9, which had suggested that the perceived time is elongated by richer encoding of memory, but not because of the perception. 
Further Analytical Implications Important to empathize in experiment 1, during deep inhalations, observers had noticed when the perceived motion of the cyclic element is decelerated, the cyclic elements are dilated (dilation mean value $\approx 0.34$, and $\mathrm{SEM} \approx 0.046$ ); and this observation is tightly consistent with our previous findings in reference 2 . This study seems to answer whether soccer players like Lionel Messi can slow the time down (see reference 11); but through their timely-controlled deep inhalations, in addition to, extraordinary spatial attention. Based on our current and previous results, timely-controlled deep inhalations, and well-positioned spatial attention do not only slow the time down, but it elongates the length which gives further advantage to the performing players to professionally deal with the dangerous tackles. Noticeably, dilation to time and length whether it's through spatial attention or deep inhalation had caused at least one activity of sympathetic nervous system activities; namely, significant dilation to time and length through spatial attention requires dilated pupils, see reference 2 ; however, significant dilation to time and length through deep inhalation triggers increased heart rates. Based on the previous notes, we might argue that extremely high activations of sympathetic nervous system might stop the perceived motion; namely, consciousness might not be updated by further dynamic conscious information; a speculation that we had previously offered "inspired by the fact that vegetative state patients are suffering from way higher heart rates than average populations", see reference 12. Interestingly to mention, one of the subjects had reported that she might be able to extremely slow the perceived motion down through extremely long deep inhalation; however, since we had been suspecting that extremely long deep inhalation might harm the human nerve system, ultimately but riskily if a subject is able to stop the perceived motion, it might enter the subject into vegetate state. We therefore had requested that nobody shall try extremely very long deep inhalation; until further neurophysiological studies are achieved. To achieve high rates of information transfer, see reference 13 , intended deep inhalation seems to be extremely important. Namely, it may allow the professional athletics to transfer high rates of rapid precise information into their surrounding environments. These fast-accurate behaviors may allow us to understand the astonishing movements exerted by Lionel Messi, namely, it could be due to the fine and detailed information gathered during his deep inhalation period; a period that triggers perception of dilated time and length.

Essential Notifications

Readers should have direct access to the stimuli, see reference 5. Advisably, download the videos for the best quality. Be sure that you have dilated pupils before the experiments, if you don't see illusory motion reversals in the experiment one's frame of reference, then you're still not ready. In order to dilate the pupil, watch the video in fifty percent brightness, and have some caffeine intake, see reference 10. During the experiment kindly follow the previously mentioned protocol, namely, take a deep inhalation, execute a profound exhalation, take an extremely very short break, and then repeat the cycle until the end of the trial! Scholars with brain imaging, ECG, and professional eye-tracker facilities are welcome to collaborate in challenging investigations, namely, to see what all of these facilities can tell us about the current experiment. Scholars who wish to acquire high temporal resolution neurophysiological signals from different nodes in $\mathrm{hMT}+$ region to distinguish between the central and the peripheral hMT+ through the present experiments are extremely welcome. Enquiries may be sent to the author. Finally, we had to confirm here that deep inhalation and exhalation had shown abilities to not only alter visual motion perception, but to sufficiently control the perceptual rivalry yields by the tested stimuli.

Acknowledgement

We would like to sincerely thank Edward Tehounik for his outstanding feedbacks and assistance.

Transactional References

[1] Yousef, A. 2019. "Inactivated Peripheral Fusiform Face Area." PsyArXiv. doi:10.31234/osf.io/u4xyz.

[2] Yousef, A. 2019. "Spatial Attention Dilates Time and Length." PsyArXiv. doi:10.31234/osf.io/qazj9.

[3] Berntson etal., (1993). Respiratory sinus arrhythmia: autonomic origins, physiological mechanisms, and psychophysiological implications. Psychophysiology.

[4] C. Ludwig, (1847). On the influence of respiratory movements on blood flow in the aortic system. Leipzig.

[5] You may find the stimuli in the following link: https://drive.google.com/drive/folders/1ErvAvZplxpHSTcCATunQK5C8PHJ8Mou

[6] Yousef, A. 2019. "Retinal Peripheries Generate Illusory Motion Reversals." PsyArXiv. doi:10.31234/osf.io/gfmpe.

[7] Thompson (1982). Perceived rate of movement depends on contrast. Vision Research.

[8] Kuriki, Ashida, Murakami, and Kitaoka, (2008). Functional brain imaging of the 'Rotating Snakes' illusion. J Vision.

[9] C. Stetson, M. Fiesta, and D. Eagleman, (2007). Does Time Really Slow Down during a Frightening Event? PLoS One.

[10] Abokyi, etal.,(2017). Caffeine intake is associated with pupil dilation and enhanced accommodation. Eye (Lond) Journal.

[11] Sajad Jafari \& Leslie Samuel Smith (2016) "Can Lionel Messi's brain slow down time passing?" Chronobiology International

[12] Yousef, A. 2019. "Rescuing Lives: When Cardiology Interweaves with Cognitive Neuroscience." PsyArXiv. doi:10.31234/osf.io/fjmy9.

[13] Tehovnik and Chen (2015). Brain control and information transfer. Experimental Brain Research. 